\title{
Numerical investigation of leaky modes in helical structural waveguides embedded into a solid medium
}

\author{
K. L. Nguyen ${ }^{\mathrm{a}}$, F. Treyssède $\mathrm{a}^{\mathrm{a}, *}$ \\ ${ }^{a}$ LUNAM Université, IFSTTAR, GERS, F-44344 Bouguenais, France
}

\begin{abstract}
Helical multi-wire cables are widely used in bridges (suspended or prestressed) and anchored retaining wall constructions. Such structures can be damaged or degraded due to corrosion and fatigue. Non destructive evaluation techniques are required to reveal defects inside cable structures. Among these numerous techniques, elastic guided waves are of potential interest owing to their ability to propagate over long distances. However in civil engineering, cables are often buried or grouted in large solid media that can be considered as unbounded. Waves can strongly attenuate along the guide axis due to the energy leakage into the surrounding medium, which reduces the propagating distance. This energy leakage can be enhanced in helical structures, which further complicates their inspection. Searching modes with low attenuation becomes necessary. The goal of this work is to propose a numerical approach to compute modes in embedded helical structures, combining the so-called semi analytical finite element method and a radial perfectly matched layer technique. Two types of radial perfeclty matched layer, centered and off-centered, are considered. Both are implemented in a twisting coordinate system which preserves translational invariance. The centered configuration is validated thanks to the twisted cylinder test case. The effect of twist on the eigenspectrum is briefly discussed. Then, an embedded helical wire of circular cross-section is considered. The off-centered configuration is shown to give the same results as the centered one. The effect of twist on modal attenuation is investigated. Finally, computations are performed for a seven-wire strand embedded into concrete, widely used in civil engineering cables.
\end{abstract}

Keywords: waveguide, helical, embedded, leaky, finite element, perfectly matched layer

\section{Introduction}

Helical structures are present in various domains, such as electromagnetism and civil engineering. A typical example in civil engineering is multi-wire cables, widely used in bridges (suspended or prestressed) and anchored retaining wall constructions. Cables can be damaged or degraded due to corrosion and fatigue. Non destructive evaluation (NDE) techniques are required to evaluate defects inside cable structures. Among these numerous techniques, elastic guided waves are of particular interest owing to their ability to propagate over long distances. Because such waves are multimodal and dispersive, modeling tools are required in practice for interpreting measurements and optimizing inspection techniques.

Due to the complexity of equations in helical systems, analytical solutions are difficult or impossible to achieve. Purely numerical approaches have to be adopted. A classical method that has been widely used for straight waveguides is the so-called semi-analytical finite element (SAFE) method. This method restricts the FE discretization to transverse directions only [1, 2, 3, 4]. It has been applied for modeling closed helical waveguides (guides in vacuum) in Ref. [5], where the SAFE modeling of a free single helical wire has been presented based on helical coordinates. A particular twisting coordinate system has then been proposed for the analysis of single helical wires as well as multi-wire strands [6].

Regardless helicity, structural waveguides are often embedded in large solid media that can be considered as unbounded. Waves can radiate energy into the surrounding medium and strongly attenuate along the guide axis, which

\footnotetext{
${ }^{*}$ Corresponding author

Email address: fabien.treyssede@ifsttar.fr (F. Treyssède)
} 
reduces the propagation distance. Such wave modes are referred to as leaky modes [7, 8]. This energy leakage can be enhanced in curved or helical structures, which makes their NDE more difficult. The curvature effect on radiation loss has been thoroughly studied in electromagnetism $[9,10,11,12,13]$ and sometimes investigated in elastodynamics $[14,15]$. In the context of NDE, searching the less attenuated modes is necessary in order to maximize the inspection distance.

As opposed to closed waveguides, the numerical modeling of embedded waveguides encounters two difficulties: the cross-section is unbounded and the amplitude of leaky modes transversely grows [16, 17, 18, 19]. In order to overcome these difficulties, the SAFE method must be combined with other numerical techniques.

As far as straight waveguides are concerned, several techniques have been recently proposed to extend the SAFE method to guides embedded in a solid matrix. A simple numerical procedure is the absorbing layer (AL) method proposed in Refs. [20, 21], which consists in creating artificial viscoelastic layers in the surrounding medium for absorbing waves. Instead of using artificial layers, Mazzotti et al. [22] have combined the boundary element method (BEM) with the SAFE method, which avoids the discretization of the unbounded surrounding domain. An alternative technique is the perfectly matched layer (PML) method. Recently, the authors have presented and analyzed SAFE-PML methods for modeling embedded solid multi-layer plates [23] and three dimensional waveguides of arbitrary cross-section [24]. These works are yet limited to straight waveguides. In electromagnetism, a SAFE-PML formulation has been proposed for the analysis of twisted microstructured optical fibers [25, 26]. Yet to the authors knowledge, the modeling of embedded helical structures has not yet been considered in elastodynamics.

The goal of this paper is to propose a SAFE-PML technique to compute leaky modes in embedded helical structures. The twisted SAFE-PML method is described in Sec. 2. The equilibrum equations of elastodynamics are written in twisting coordinates to account for the helical geomtery. In this coordinate system, a radial PML is applied. This radial PML can be centered or off-centered. The method is validated in Sec. 3 thanks to the cylindrical bar test case, which can support any arbitrary twist. The effect of twist on the eigenspectrum is briefly discussed. Two numerical applications are then presented in Sec. 4. The first example consists in studying an embedded helical wire of circular cross-section. The effect of twist on the axial attenuation of modes is investigated. The second application is a seven-wire strand embedded into concrete. Seven-wire strands are widely used in civil engineering cables. They are typically made by one straight cylindrical wire surrounded by one layer of six helical wires.

\section{Numerical method}

\subsection{Elastodynamics in twisting coordinates}

Let us introduce a twisting coordinate system $(x, y, z)$ defined from the Cartesian coordinates $(X, Y, Z)[6]$ :

$$
\begin{aligned}
& x=X \cos (\tau Z)+Y \sin (\tau Z) \\
& y=-X \sin (\tau Z)+Y \cos (\tau Z) \\
& z=Z
\end{aligned}
$$

where $\tau$ denotes the torsion, which characterizes the rotation rate of the $(x, y)$ plane along the $z$ axis. In the Cartesian basis ( $\left.\mathbf{e}_{X}, \mathbf{e}_{Y}, \mathbf{e}_{Z}\right)$, the twisting basis $\left(\mathbf{e}_{x}, \mathbf{e}_{y}, \mathbf{e}_{z}\right)$ is expressed as follows:

$$
\begin{aligned}
& \mathbf{e}_{x}=\cos (\tau Z) \mathbf{e}_{X}+\sin (\tau Z) \mathbf{e}_{Y} \\
& \mathbf{e}_{y}=-\sin (\tau Z) \mathbf{e}_{X}+\cos (\tau Z) \mathbf{e}_{Y} \\
& \mathbf{e}_{z}=\mathbf{e}_{Z}
\end{aligned}
$$

One considers a three-dimensional helical waveguide $\tilde{\Omega}=\tilde{S} \times]-\infty,+\infty[$ whose cross-section $\tilde{S}$ lies in the transverse $(\tilde{x}, \tilde{y})$ plane and is invariant along the $z$ axis. The tilde notation will be explained through the introduction of PML in Sec. 2.2.

The time harmonic dependence is chosen as $e^{-i \omega t}$. Linear elastic materials are assumed. As this study focuses on eigenmodes, acoustic sources and external forces are discarded. In the twisting coordinate system, the threedimensional variational formulation of elastodynamics is given by [6]:

$$
\int_{\tilde{\Omega}} \delta \tilde{\boldsymbol{\epsilon}}^{T} \tilde{\boldsymbol{\sigma}} d \tilde{\Omega}-\omega^{2} \int_{\tilde{\Omega}} \tilde{\rho} \delta \tilde{\mathbf{u}}^{T} \tilde{\mathbf{u}} d \tilde{\Omega}=0
$$




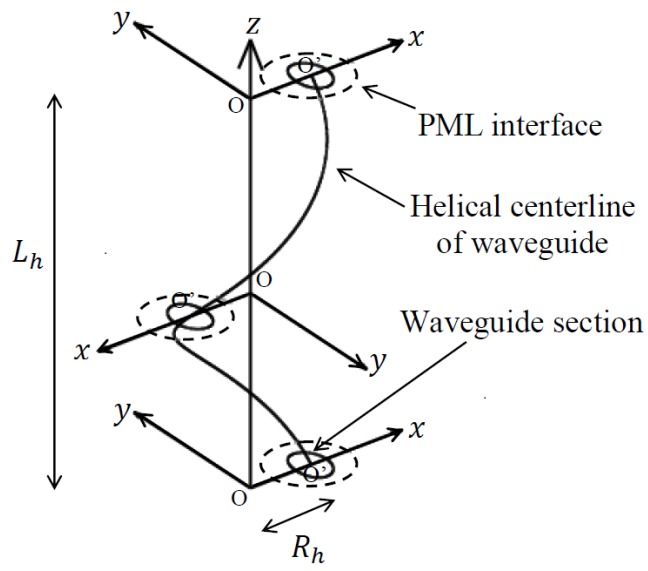

(a)

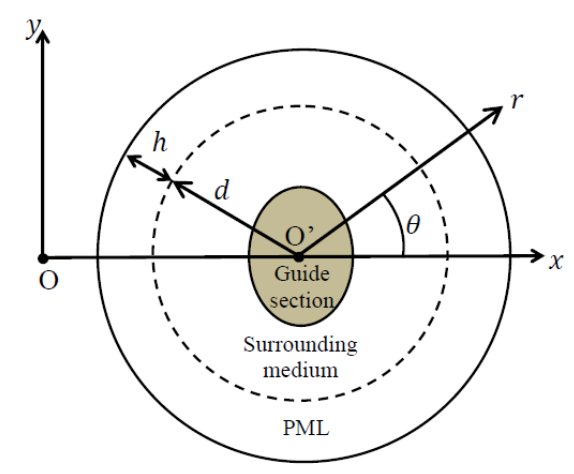

(b)

Figure 1: (a) Off-centered twisting cylindrical coordinates $\left(x_{O^{\prime}} \neq 0, y_{O^{\prime}}=0\right)$, (b) Truncated section with an offcentered PML.

where $d \tilde{\Omega}=d \tilde{x} d \tilde{y} d z$. The strain-displacement relation is:

$$
\tilde{\boldsymbol{\epsilon}}=\left(\mathbf{L}_{\tilde{S}}+\mathbf{L}_{z} \frac{\partial}{\partial z}\right) \tilde{\mathbf{u}}
$$

where the operators separating transverse from axial derivatives are:

$$
\mathbf{L}_{\tilde{S}}=\left[\begin{array}{ccc}
\partial / \partial \tilde{x} & 0 & 0 \\
0 & \partial / \partial \tilde{y} & 0 \\
0 & 0 & \Lambda_{\tilde{S}} \\
\partial / \partial \tilde{y} & \partial / \partial \tilde{x} & 0 \\
\Lambda_{\tilde{S}} & -\tau & \partial / \partial \tilde{x} \\
\tau & \Lambda_{\tilde{S}} & \partial / \partial \tilde{y}
\end{array}\right], \quad \mathbf{L}_{z}=\left[\begin{array}{ccc}
0 & 0 & 0 \\
0 & 0 & 0 \\
0 & 0 & 1 \\
0 & 0 & 0 \\
1 & 0 & 0 \\
0 & 1 & 0
\end{array}\right]
$$

with $\Lambda_{\tilde{S}}=\tau \tilde{y} \frac{\partial}{\partial \tilde{x}}-\tau \tilde{x} \frac{\partial}{\partial \tilde{y}}$.

The formulation (3) holds for any kinematically admissible displacement $\delta \tilde{\mathbf{u}}=\left[\delta \tilde{u}_{x} \delta \tilde{u}_{y} \delta \tilde{u}_{z}\right]^{T}$. The superscript

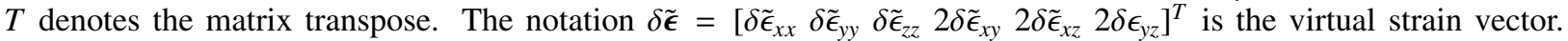
Similarly, $\tilde{\boldsymbol{\sigma}}=\left[\begin{array}{llllll}\tilde{\sigma}_{x x} & \tilde{\sigma}_{y y} & \tilde{\sigma}_{z z} & \tilde{\sigma}_{x y} & \tilde{\sigma}_{x z} & \tilde{\sigma}_{y z}\end{array}\right]^{T}$ denotes the stress vector. Vector components are expressed in the twisting basis $\left(\mathbf{e}_{x}, \mathbf{e}_{y}, \mathbf{e}_{z}\right)$. The stress-strain relation is given by $\tilde{\boldsymbol{\sigma}}=\tilde{\mathbf{C}} \tilde{\boldsymbol{\epsilon}}$, where $\tilde{\mathbf{C}}$ is the matrix of material properties. $\tilde{\rho}$ is the material mass density. We assume that $\tilde{\mathbf{C}}$ and $\tilde{\rho}$ depend only on the twisting transverse coordinates $(\tilde{x}, \tilde{y})$, which means that the problem is translationally invariant along the $z$ axis.

\subsection{Radial $P M L$}

Let us define the cylindrical representation $(r, \theta, z)$ of the twisting coordinates $(x, y, z)$ from: $\tilde{x}=x_{O^{\prime}}+\tilde{r} \cos \theta$, $\tilde{y}=y_{O^{\prime}}+\tilde{r} \sin \theta$. In the $(x, y)$ plane, the point $O^{\prime}$ of coordinates $\left(x_{O^{\prime}}, y_{O^{\prime}}\right)$ is the center of this cylindrical system. $x_{O^{\prime}}$ and $y_{O^{\prime}}$ are independent of the axial coordinate $z$. As shown by Fig. 1a, the point $O^{\prime}$ thus defines a helix as it travels in the $z$ direction. A helix curve can be characterized by two parameters: $R_{h}$, the helix radius in the $(x, y)$ plane and $L_{h}$, the helix step along the $z$ axis. The torsion of the twisting coordinate system attached to the helix is defined by $\tau=2 \pi / L_{h}[6]$. In the remainder of this paper, we will set $y_{O^{\prime}}=0$ without loss of generality. 


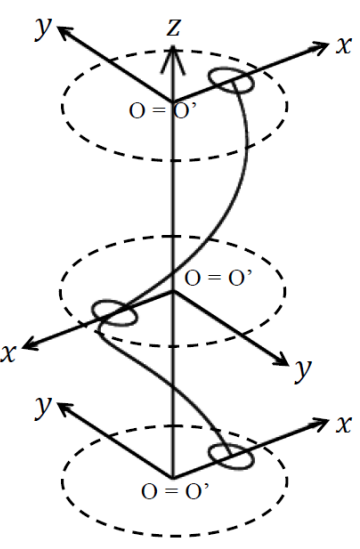

(a)

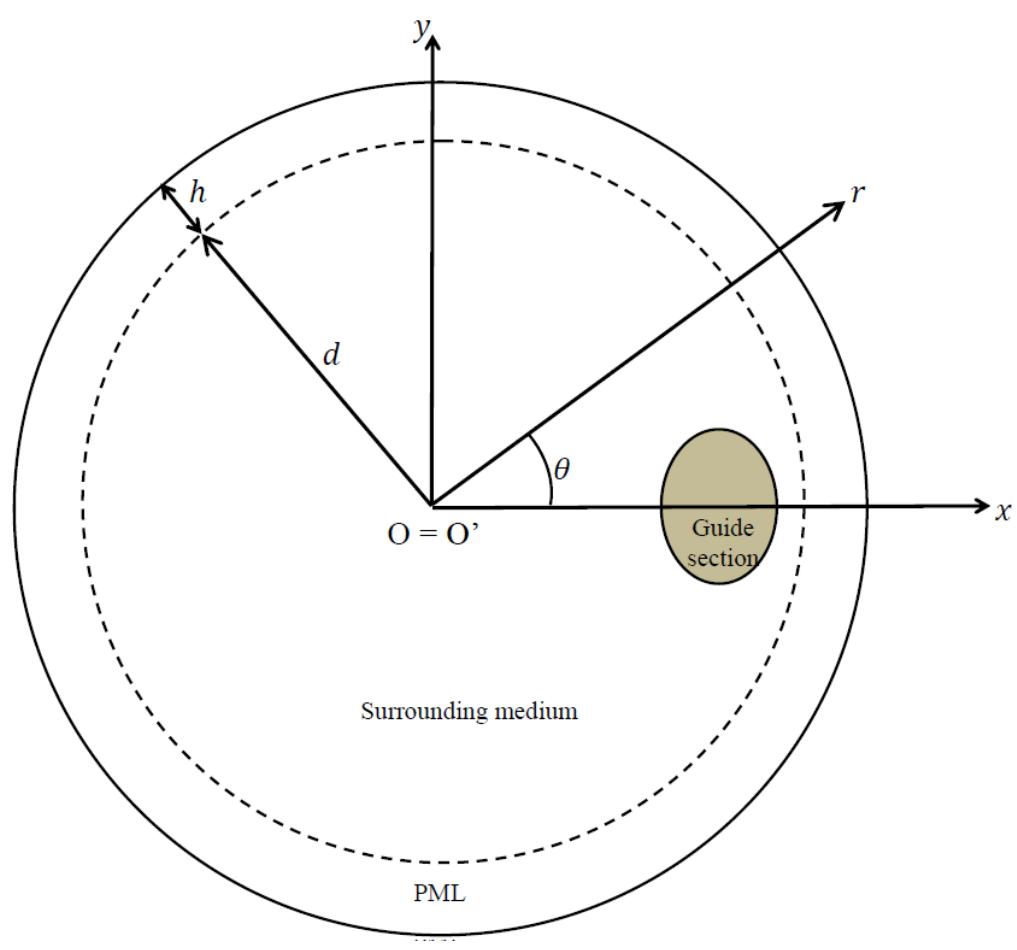

(b)

Figure 2: (a) Centered twisting cylindrical coordinates $\left(x_{O^{\prime}}=y_{O^{\prime}}=0\right)$, (b) Truncated section with a centered PML.

For clarity, the Jacobian matrix of the transformation from $(\tilde{x}, \tilde{y}, z)$ to $(\tilde{r}, \theta, z)$ and its inverse are given by:

$$
\begin{aligned}
\mathbf{J} & =\left[\begin{array}{lll}
\partial \tilde{x} / \partial \tilde{r} & \partial \tilde{x} / \partial \theta & \partial \tilde{x} / \partial z \\
\partial \tilde{y} / \partial \tilde{r} & \partial \tilde{y} / \partial \theta & \partial \tilde{y} / \partial z \\
\partial z / \partial \tilde{r} & \partial z / \partial \theta & \partial z / \partial z
\end{array}\right]=\left[\begin{array}{ccc}
\cos \theta & -\tilde{r} \sin \theta & 0 \\
\sin \theta & \tilde{r} \cos \theta & 0 \\
0 & 0 & 1
\end{array}\right], \\
\mathbf{J}^{-1} & =\left[\begin{array}{lll}
\partial \tilde{r} / \partial \tilde{x} & \partial \tilde{r} / \partial \tilde{y} & \partial \tilde{r} / \partial z \\
\partial \theta / \partial \tilde{x} & \partial \theta / \partial \tilde{y} & \partial \theta / \partial z \\
\partial z / \partial \tilde{x} & \partial z / \partial \tilde{y} & \partial z / \partial z
\end{array}\right]=\frac{1}{\tilde{r}}\left[\begin{array}{ccc}
\tilde{r} \cos \theta & \tilde{r} \sin \theta & 0 \\
-\sin \theta & \cos \theta & 0 \\
0 & 0 & \tilde{r}
\end{array}\right]
\end{aligned}
$$

The formulation (3) is now transformed into cylindrical coordinates, but with vectors and tensors still expressed in the basis $\left(\mathbf{e}_{x}, \mathbf{e}_{y}, \mathbf{e}_{z}\right)$. One has $d \tilde{\Omega}=\tilde{r} d \tilde{r} d \theta d z$. The operator $\mathbf{L}_{z}$ of the strain-displacement relation (4) is unchanged. The operator $\mathbf{L}_{\tilde{S}}$ is rewritten, thanks to the Jacobian matrix $\mathbf{J}^{-1}$ in Eq. (6), by replacing $\partial / \partial \tilde{x}, \partial / \partial \tilde{y}$ and $\Lambda_{\tilde{S}}$ with:

$$
\frac{\partial}{\partial \tilde{x}}=\cos \theta \frac{\partial}{\partial \tilde{r}}-\frac{\sin \theta}{\tilde{r}} \frac{\partial}{\partial \theta}, \frac{\partial}{\partial \tilde{y}}=\sin \theta \frac{\partial}{\partial \tilde{r}}+\frac{\cos \theta}{\tilde{r}} \frac{\partial}{\partial \theta}, \Lambda_{\tilde{S}}=-\tau x_{O^{\prime}} \sin \theta \frac{\partial}{\partial \tilde{r}}-\tau\left(x_{O^{\prime}} \frac{\cos \theta}{\tilde{r}}+1\right) \frac{\partial}{\partial \theta} .
$$

The main difficulty for modeling an embedded waveguide is the unbounded nature of its cross-section. The basic idea proposed in this paper consists in closing the cross-section thanks to a PML introduced along the transverse directions, in the exterior domain to absorb waves. The unbounded section is hence truncated at a finite distance. In this paper, $\tilde{S}$ denotes the truncated section including PML. The tilde notation used throughout this paper means that a PML has been introduced along the transverse direction.

The PML technique for open waveguides consists in transforming real transverse coordinates [23, 24, 25] into complex ones. With a PML applied in the radial direction, the formulation (3) can then be interpreted as the analytical 
continuation of equilibrium equations into the complex radial coordinate $\tilde{r}$ defined as:

$$
\tilde{r}(r)=\int_{0}^{r} \gamma(\xi) d \xi
$$

where $\gamma(r)$ is a complex function satisfying:

- $\gamma(r)=1$ for $r \leq d$

- $\operatorname{Im}\{\gamma\}>0$ for $r>d$

$d$ is the radius of the PML interface. In this paper, a twisted radial PML will be called off-centered if $x_{O^{\prime}} \neq 0$ and centered if $x_{O^{\prime}}=0$ (see Figs. 1a and 2a respectively). The centered PML is of particular interest and will be discussed later in Secs. 2.4 and 3. The main advantage of the off-centered PML is that the computational domain can be greatly reduced compared to the centered PML, as shown later in Sec. 4.1 for the analysis of a single helical wire.

As shown in Figs. $1 b$ and $2 b$, the whole cross-section of the problem (including PML) is typically a circle of radius $d+h$. On the exterior boundary of the PML, the boundary condition can be arbitrarily chosen (usually of Dirichlet type).

From Eq. (8), the change of variables $\tilde{r} \mapsto r$ yields for any function $\tilde{f}$ :

$$
\frac{\partial \tilde{f}}{\partial \tilde{r}}=\frac{1}{\gamma} \frac{\partial f}{\partial r}, d \tilde{r}=\gamma d r
$$

where $\tilde{f}(\tilde{r}, \theta, z)=f(r, \theta, z)$. Applying this change of variables, the formulation (3) can be rewritten in real radial coordinates. One has $d \tilde{\Omega}=\tilde{r} \gamma / r d x d y d z$. Following Eqs. (7) and (9), the operator $\mathbf{L}_{\tilde{S}}$ given by Eq. (5) is transformed by replacing $\partial / \partial \tilde{x}, \partial / \partial \tilde{y}$ and $\Lambda_{\tilde{S}}$ with:

$$
\frac{\partial}{\partial \tilde{x}}=\frac{\cos \theta}{\gamma} \frac{\partial}{\partial r}-\frac{\sin \theta}{\tilde{r}} \frac{\partial}{\partial \theta}, \frac{\partial}{\partial \tilde{y}}=\frac{\sin \theta}{\gamma} \frac{\partial}{\partial r}+\frac{\cos \theta}{\tilde{r}} \frac{\partial}{\partial \theta}, \quad \Lambda_{\tilde{S}}=-\frac{\tau}{\gamma} x_{O^{\prime}} \sin \theta \frac{\partial}{\partial r}-\tau\left(x_{O^{\prime}} \frac{\cos \theta}{\tilde{r}}+1\right) \frac{\partial}{\partial \theta} .
$$

Finally, the variational formulation and the strain-displacement relation must be transformed back to the initial twisting coordinates $(x, y, z)$. Thanks to the Jacobian matrix $\mathbf{J}$ of Eq. (6), replacing $\cos \theta$ and $\sin \theta$ by $\left(x-x_{O^{\prime}}\right) / r$ and $y / r$ respectively, the derivatives with respect to $(r, \theta)$ can be expressed in terms of $(x, y)$ as follows:

$$
\frac{\partial}{\partial r}=\frac{x-x_{O^{\prime}}}{r} \frac{\partial}{\partial x}+\frac{y}{r} \frac{\partial}{\partial y}, \frac{\partial}{\partial \theta}=-y \frac{\partial}{\partial x}+\left(x-x_{O^{\prime}}\right) \frac{\partial}{\partial y} .
$$

From Eqs. (10) and (11), the initial operator $\mathbf{L}_{\tilde{S}}$ is finally transformed into the following operator, denoted as $\mathbf{L}_{S}$ :

$$
\mathbf{L}_{S}=\left[\begin{array}{ccc}
\Lambda_{x} & 0 & 0 \\
0 & \Lambda_{y} & 0 \\
0 & 0 & \Lambda_{S} \\
\Lambda_{y} & \Lambda_{x} & 0 \\
\Lambda_{S} & -\tau & \Lambda_{x} \\
\tau & \Lambda_{S} & \Lambda_{y}
\end{array}\right]
$$

with the notations:

$$
\begin{array}{r}
\Lambda_{x}=\left(\frac{\left(x-x_{O^{\prime}}\right)^{2}}{\gamma r^{2}}+\frac{y^{2}}{\tilde{r} r}\right) \frac{\partial}{\partial x}+\left(\frac{1}{\gamma r^{2}}-\frac{1}{\tilde{r} r}\right)\left(x-x_{O^{\prime}}\right) y \frac{\partial}{\partial y}, \Lambda_{y}=\left(\frac{1}{\gamma r^{2}}-\frac{1}{\tilde{r} r}\right)\left(x-x_{O^{\prime}}\right) y \frac{\partial}{\partial x}+\left(\frac{y^{2}}{\gamma r^{2}}+\frac{\left(x-x_{O^{\prime}}\right)^{2}}{\tilde{r} r}\right) \frac{\partial}{\partial y} \\
\Lambda_{S}=\tau\left[-\frac{x_{O^{\prime}}}{\gamma} \frac{y\left(x-x_{O^{\prime}}\right)}{r^{2}}+\left(x_{O^{\prime}} \frac{x-x_{O^{\prime}}}{r \tilde{r}}+1\right) y\right] \frac{\partial}{\partial x}-\tau\left[\frac{x_{O^{\prime}} y^{2}}{\gamma r^{2}}+\left(x_{O^{\prime}} \frac{x-x_{O^{\prime}}}{r \tilde{r}}+1\right)\left(x-x_{O^{\prime}}\right)\right] \frac{\partial}{\partial y}
\end{array}
$$

As in any problem involving PML, the profile of the function $\gamma$ influences the accuracy of numerical results. For modeling embedded waveguides, this PML function has been usually set to a constant complex value [27, 28, 29, 26], yielding a discontinuity at the PML interface. Due to FE discretization, this discontinuity may yield non negligible 
reflection. As shown in Refs. [23, 30], a smooth profile can improve the accuracy. In this paper, a parabolic function is set for both the real and the imaginary parts of $\gamma$ :

$$
\gamma(r)=\left\{\begin{array}{lll}
1 & \text { if } & r \leq d \\
1+3(\hat{\gamma}-1)\left(\frac{r-d}{h}\right)^{2} & \text { if } & r>d
\end{array}\right.
$$

where $\hat{\gamma}$ is the average value of $\gamma$ inside the PML region:

$$
\hat{\gamma}=\frac{1}{h} \int_{d}^{d+h} \gamma(\xi) d \xi
$$

The value $\hat{\gamma}$ hence quantifies the PML absorption [23].

\subsection{SAFE technique}

The SAFE method consists in assuming the harmonic dependence of fields in the axial direction $z$ before FE discretization, which has the advantage to reduce the three dimensional problem to the two dimensional cross-section of the waveguide.

Applying the SAFE technique, the displacement $\mathbf{u}$ and the virtual one $\delta \mathbf{u}$ are then expressed on one finite element $e$ as follows:

$$
\mathbf{u}(x, y, z)=\mathbf{N}^{e}(x, y) \mathbf{U}^{e} e^{i k z}, \delta \mathbf{u}(x, y, z)=\mathbf{N}^{e}(x, y) \delta \mathbf{U}^{e} e^{-i k z}
$$

where $k$ denotes the axial wavenumber, $\mathbf{U}^{e}$ and $\delta \mathbf{U}^{e}$ are the nodal displacement vectors and $\mathbf{N}^{e}$ is the matrix of interpolating functions on the element $e$.

Replacing the axial derivative $\partial / \partial z$ of the trial and test functions with products by $+i k$ and $-i k$ respectively, the formulation (3) is reduced from three dimensions $(x, y, z)$ to a bidimensional problem written in the transverse directions $(x, y)$. The strain-displacement relation (4) and the virtual one become:

$$
\boldsymbol{\epsilon}=\left(\mathbf{L}_{S}+i k \mathbf{L}_{z}\right) \mathbf{N}^{e} \mathbf{U}^{e} e^{i k z}, \delta \boldsymbol{\epsilon}=\left(\mathbf{L}_{S}-i k \mathbf{L}_{z}\right) \delta \mathbf{N}^{e} \mathbf{U}^{e} e^{-i k z}
$$

The FE discretization of the cross-section yields finally:

$$
\left\{\mathbf{K}_{1}-\omega^{2} \mathbf{M}+i k\left(\mathbf{K}_{2}-\mathbf{K}_{2}^{T}\right)+k^{2} \mathbf{K}_{3}\right\} \mathbf{U}=\mathbf{0}
$$

with the elementary matrices:

$$
\begin{aligned}
\mathbf{K}_{1}^{e} & =\int_{e} \mathbf{N}^{e T} \mathbf{L}_{\tilde{S}}^{T} \mathbf{C} \mathbf{L}_{\tilde{S}} \mathbf{N}^{e} \frac{\gamma \tilde{r}}{r} d x d y, \mathbf{K}_{2}^{e}=\int_{e} \mathbf{N}^{e T} \mathbf{L}_{\tilde{S}}^{T} \mathbf{C} \mathbf{L}_{z} N^{e} \frac{\gamma \tilde{r}}{r} d x d y \\
\mathbf{K}_{3}^{e} & =\int_{e} \mathbf{N}^{e T} \mathbf{L}_{z}^{T} \mathbf{C} \mathbf{L}_{z} \boldsymbol{N}^{e} \frac{\gamma \tilde{r}}{r} d x d y, \mathbf{M}^{e}=\int_{e} \rho \mathbf{N}^{e T} \mathbf{N}^{e} \frac{\gamma \tilde{r}}{r} d x d y
\end{aligned}
$$

Note that the SAFE-PML matrices are complex due to the functions $\gamma$ and $\tilde{r}$ in the integrands.

Given the frequency $\omega$, the formulation (18) corresponds to a quadratic eigenproblem with respect to $k$, which can be transformed into the following linear form:

$$
(\mathbf{A}-k \mathbf{B}) \hat{\mathbf{U}}=0
$$

with :

$$
\mathbf{A}=\left[\begin{array}{cc}
\mathbf{0} & \mathbf{I} \\
-\left(\mathbf{K}_{1}-\omega^{2} \mathbf{M}\right) & -i\left(\mathbf{K}_{2}-\mathbf{K}_{2}^{T}\right)
\end{array}\right], \quad \mathbf{B}=\left[\begin{array}{cc}
\mathbf{I} & \mathbf{0} \\
\mathbf{0} & \mathbf{K}_{3}
\end{array}\right], \quad \hat{\mathbf{U}}=\left[\begin{array}{c}
\mathbf{U} \\
k \mathbf{U}
\end{array}\right]
$$

In this paper, the ARPACK library [31] is used for solving the eigensystem (19). This library is based on an implicitly restarted Arnoldi method. For each frequency, a specified number of eigenvalues is looked for around a user-defined shift.

Note that the symmetry of $\mathbf{K}_{1}, \mathbf{K}_{3}$ and $\mathbf{M}$ implies that if $k$ is an eigenvalue of (18), then $-k$ is also an eigenvalue. Thus, the eigenspectrum includes two families of solutions, $\left(k_{j}, \mathbf{U}_{j}^{+}\right)$and $\left(-k_{j}, \mathbf{U}_{j}^{-}\right),(j=1, \ldots, n)$ representing $n$ positive-going and $n$ negative-going waves. 


\subsection{Case of centered PML}

For the centered configuration $\left(x_{O^{\prime}}=0\right)$, the radial PML centerline is the $z$-axis (see Fig. 2). As shown below, this particular case is of interest because it allows to justify the absorption of waves inside a twisted PML thanks to analytical wave solutions. Such a PML has been proposed for modeling embedded optical fibers in Refs. [25, 26].

Let us define the untwisting cylindrical coordinates $(\tilde{R}, \Theta, Z)$ from the Cartesian coordinates $(\tilde{X}, \tilde{Y}, Z): \tilde{X}=$ $\tilde{R} \cos \Theta, \tilde{Y}=\tilde{R} \sin \Theta$. The relation between twisting and untwisting cylindrical coordinates can be deduced from Eq. (1) and yields:

$$
\tilde{r}=\tilde{R}, \quad \theta=\Theta-\tau Z, \quad z=Z
$$

One assumes negligible reflection from the exterior boundary of the truncated PML. In the untwisting coordinate system, the radial dependence of wave fields in the PML region $(R>d)$ is given by $H_{n}^{(1)}\left(k_{r} \tilde{R}(R)\right)$, where $H_{n}^{(1)}$ is the Hankel function of the first kind and $k_{r}$ denotes the radial wavenumber (shear or longitudinal). According to the relation (21), this radial dependence can be rewritten in twisting coordinates as $H_{n}^{(1)}\left(k_{r} \tilde{r}(r)\right)$. Therefore for the centered configuration, a radial PML with twist will act on wave absorption exactly in the same way as the standard radial PML without twist, which has been thoroughly studied and validated in the literature [32, 33].

The particular case of a centered PML can thus be considered as the reference solution in order to validate the off-centered configuration. A numerical comparison between centered and off-centered PML will be performed in Sec. 4.1.

\subsection{Modal filtering}

As found for straight waveguides [23, 24], the SAFE-PML method provides many radiation modes in addition to the modes of interest (i.e. leaky modes, in this paper). Since radiation modes mainly oscillate inside the PML, they are strongly dependent on PML parameters: PML radiation modes cannot be considered as intrinsic to the physics and are of no interest for NDE. However, a large number of radiation modes prevents a clear visualization of the dispersion curves of leaky modes. A modal filtering technique must be post-processed to identify and remove these radiation modes from the visualization.

Following previous works for straight waveguides [23, 24], the filtering criterion proposed in this paper is based on the ratio of kinetic energy inside the PML region over the kinetic energy of the whole cross-section. Physical modes are then identified if this ratio is smaller than a user-defined value $\rho_{\max }$ :

$$
\frac{\left|E_{\mathrm{PML}}\right|}{\left|E_{\mathrm{tot}}\right|}<\rho_{\max }
$$

where $E_{\mathrm{tot}}$ and $E_{\mathrm{PML}}$ denote the modal kinetic energy of the whole cross-section $(0<r<d+h)$ and the modal kinetic energy restricted to the PML region $(d<r<d+h)$. Note that the modulus must be used in Eq. (22) since the kinetic energy is complex due to the presence of PML. One points out that the computation of kinetic energy can be readily performed from SAFE matrices and is similar to straight waveguides (see Ref. [6] for instance).

\section{Validation}

In order to validate the SAFE-PML method with twist, an embedded cylindrical bar is considered. Although the geometry is straight, such a test case supports any twist because the cross-section of a cylinder is circular in the $(x, y)$ plane and centered at $O$ (Fig. 3a). In other words, a twisted cylinder remains a straight cylinder for any value of the torsion $\tau$. Results obtained with $\tau \neq 0$ can then be checked with the reference solution obtained without twist $(\tau=0)$.

\subsection{Test case description}

One considers a steel cylinder of radius $a=10 \mathrm{~mm}$ buried in concrete. The material characteristics are given in Tab. 1. This test case is taken from the paper of Castaings et al. [20].

The PML thickness is equal to $h=0.9 a$. Following the suggestions of Refs. [32, 27, 23], the PML interface is set close to the core to reduce the effects of transverse growth of leaky modes on numerical results $(d=1.1 a)$. The cross-section of the steel-concrete cylindrical waveguide using PML is sketched in Fig. 3b. A Dirichlet condition is applied at the exterior boundary of the PML. Finite elements are six-node triangles (Fig. 3c) whose average length $l_{e}$ 


\begin{tabular}{lccc}
\hline \hline Material & $c_{l}(\mathrm{~m} / \mathrm{s})$ & $c_{s}(\mathrm{~m} / \mathrm{s})$ & $\rho\left(\mathrm{kg} / \mathrm{m}^{3}\right)$ \\
\hline Steel & 5960 & 3260 & 7932 \\
Concrete & 4222.1 & 2637.5 & 2300 \\
\hline \hline
\end{tabular}

Table 1: Material characteristics.

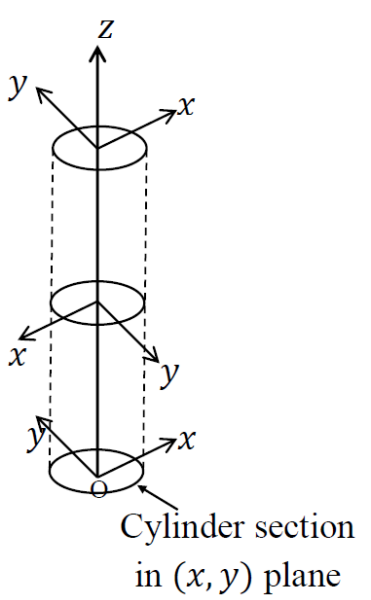

(a)

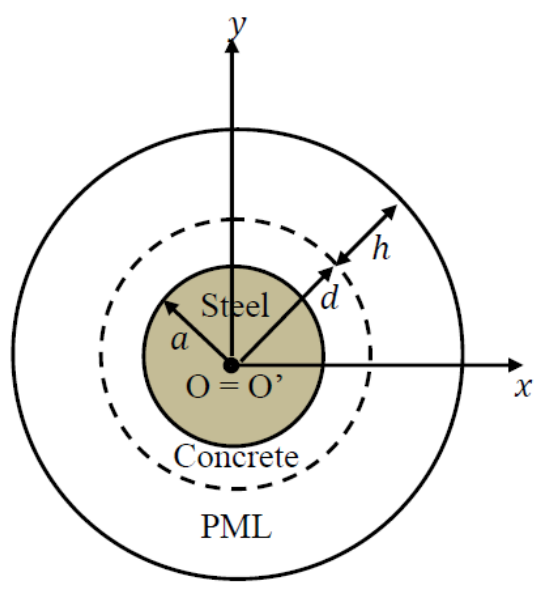

(b)

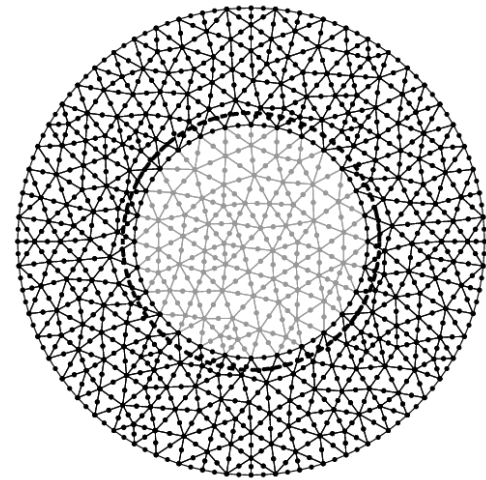

(c)

Figure 3: a) Cross-section of a cylinder in the twisting $(x, y)$ plane, b) cross-section of an embedded cylindrical bar, c) FE mesh (gray: steel core, black: embedding medium including PML). Dashed line: PML interface.

is set to $0.2 a$, satisfying the meshing criterion: $l_{e} \leq \lambda_{m} / 5$ where $\lambda_{m}=c_{s} / \max (f)$ and $c_{s}$ is the bulk shear velocity in the surrounding medium (concrete). 3975 dofs (degrees of freedom) have been generated.

At each frequency, 150 modes have been solved around the positive eigenvalue shift $+\omega / c_{l}$, where $c_{l}$ denotes the bulk longitudinal velocity in the surrounding medium. In this way, only modes of positive phase velocity are calculated.

\subsection{Results}

In order to outline the twist effect on axial wavenumbers, the SAFE-PML eigenspectrum is first briefly examined. Figure 4 a compares the spectrum of axial wavenumbers $k a$ computed by the SAFE-PML method with twist for the arbitrary torsion value $\tau a=0.134$ with the reference spectrum, obtained without twist $(\tau a=0)$. Results are presented for the dimensionless frequency $\omega a / c_{s 0}=1.5$, where $c_{s 0}$ denotes the shear velocity of the core (steel).

As shown in Fig. 4a, the SAFE-PML method does not only compute leaky modes but also gives many radiation modes. These radiation modes correspond to standing waves mainly oscillating in the PML region. As an example, Fig. 5 depicts the axial displacement of a leaky mode and of a radiation mode. Without twist, radiation modes belong to two spectra of origin $\left(\omega / c_{l}, 0\right)$ and $\left(\omega / c_{s}, 0\right)$. As explained in Refs. [23, 24], these modes correspond to the discretization of two continua associated with compressional and shear waves respectively. With twist, the SAFEPML eigenspectrum appears to be spread over a larger region than without twist. This phenomenon will be explained below.

For a clear visualisation of leaky modes, radiation modes must be filtered out by the criterion defined in Sec. 2.5. Figure $4 \mathrm{~b}$ shows the eigenspectrum obtained after modal filtering. The remaining modes corresponds to leaky modes, which can be identified by inspecting their mode shapes. In Fig. 4b, the same labeling of modes as in Ref. [34] is adopted: $L(0, m)$ for compressional modes, $T(0, m)$ for torsional modes and $F(n, m)$ for flexural modes, where $n$ denotes the circumferential order and $m$ is the radial index. 


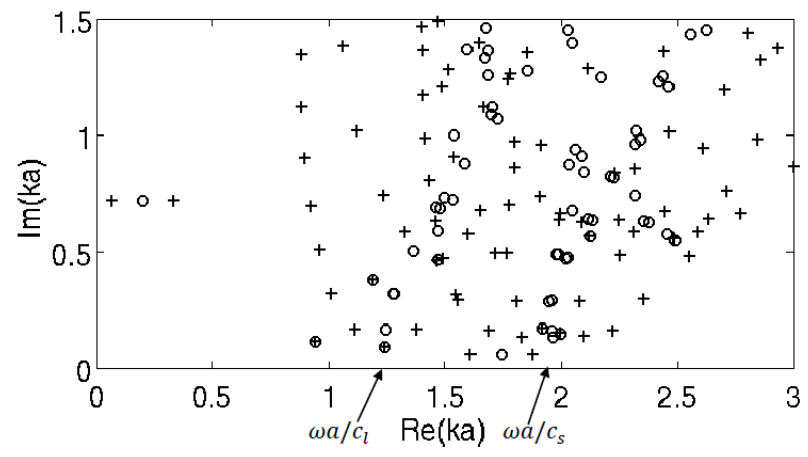

(a)

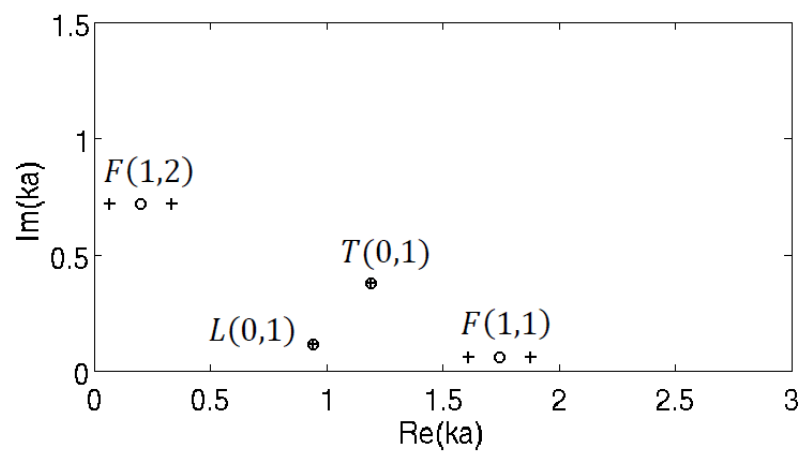

(b)

Figure 4: Spectrum at $\omega a / c_{s 0}=1.5$ of the steel-concrete waveguide computed by the SAFE-PML method with $d=1.1 a, h=0,9 a, l_{e}=0.2 a, \hat{\gamma}=2+4 i$, (a) without filter and (b) after modal filtering of radiation modes $\left(\rho_{\max }=0.9\right)$. Crosses: results for the twisting system $(\tau a=0.134)$, circles: reference results $(\tau a=0)$.

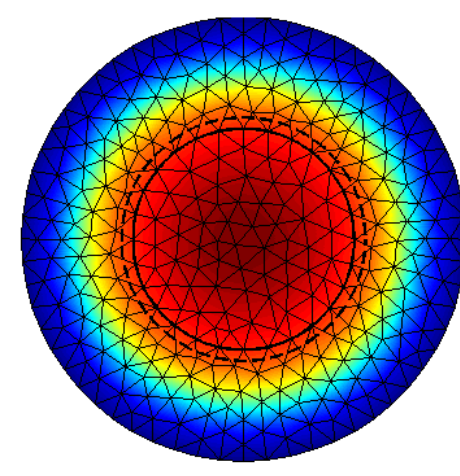

(a)

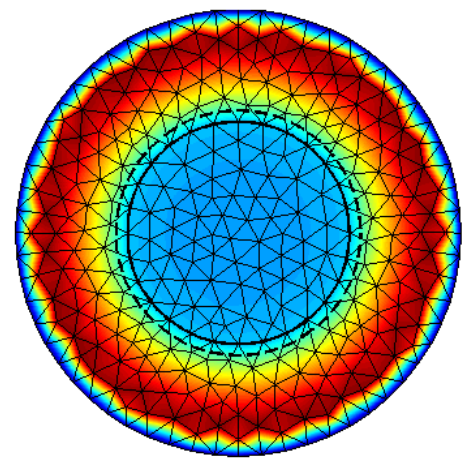

(b)

Figure 5: Modulus of axial displacement of (a) a leaky mode (the $L(0,1)$ mode) and (b) of a radiation mode at $\omega a / c_{s 0}=1.5$.

It can be observed that the wavenumbers of axisymmetric modes, i.e. compressional $L(0,1)$ and torsional $T(0,1)$ modes, are left unchanged by the twist. However, the wavenumbers of flexural modes $F(n, m)$, which are nonaxisymmetric, turn out to be different in twisting coordinates. Without twist $(\tau a=0)$, it is well-known that the axial wavenumbers of $F(m, n)$ occur in pairs of double roots [34]. With twist, these roots indeed become distinct due to the rotation of the $(x, y)$ plane around the $Z$ axis.

This phenomenon, which has already been found for closed waveguides [6,35], can be explained analytically by expressing the dependence of wave solutions in both cylindrical systems (untwisting and twisting). Let us denote the axial wavenumbers in the untwisting and twisting systems by $K$ and $k$ respectively. For leaky modes (diverging waves), the dependence of wave fields in terms of the untwisting cylindrical coordinates is in $H_{n}^{(1)}\left(k_{r} R\right) e^{ \pm i n \Theta} e^{i K Z}$. Inside the PML region, these solutions are rewritten in complex coordinates as $H_{n}^{(1)}\left(k_{r} \tilde{R}(R)\right) e^{ \pm i n \Theta} e^{i K Z}$. Thanks to Eq. (21), the dependence of wave fields can be readily rewritten as a function of twisting cylindrical coordinates, in $H_{n}^{(1)}\left(k_{r} \tilde{r}(r)\right) e^{ \pm i n \theta} e^{i(K \pm \tau n) z}$. This implies that:

$$
k=K \pm \tau n .
$$

With twist, the axial wavenumbers of leaky modes are therefore translated by \pm tna.

The translation relation (23) also holds for radiation modes because the geometry of the whole cross-section is 


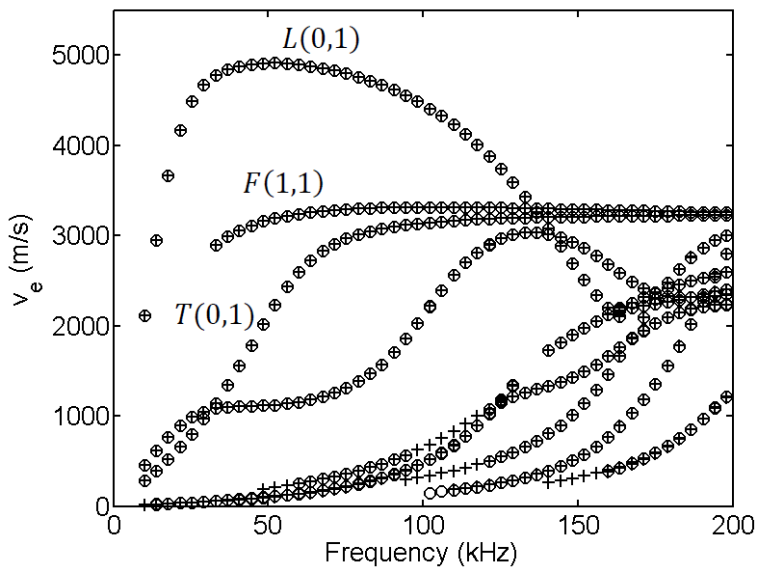

(a)

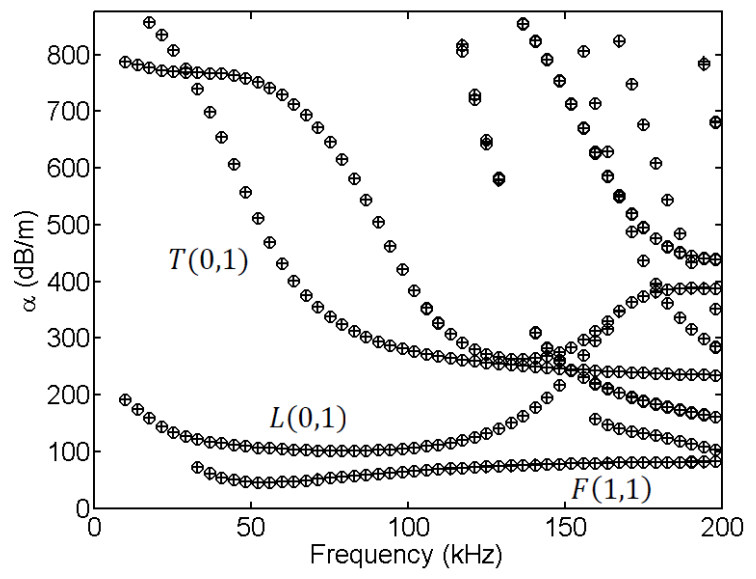

(b)

Figure 6: Energy velocity dispersion curves of the steel-concrete waveguide computed by the SAFE-PML method $\left(d=1.1 a, h=0,9 a, l_{e}=0.2 a\right)$, obtained after modal filtering $\left(\rho_{\max }=0.75\right)$. Crosses: results for the twisting system $(\tau a=0.134)$, circles: reference results $(\tau a=0)$.

circular. This explains why the eigenspectrum of radiation modes extends to a larger region than without twist (see Fig.4a). Compared to leaky modes, note that the radial dependence of radiation modes is a linear combination of Hankel functions of the first and of the second kind (diverging and converging waves).

Figure 6 shows the energy velocity dispersion curves as well as the axial attenuation in $\mathrm{dB} / \mathrm{m}$ (equal to $8.686 \operatorname{Im}(k)$ ) obtained from the twisting and the untwisting systems. It can be observed that the curves computed in both systems

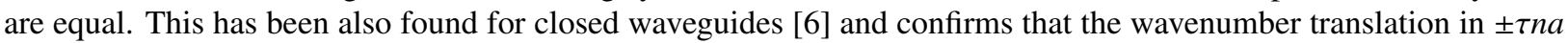
does not change the physical nature of modes in cylinders.

One points out that the dispersion curves shown in Fig. 6 are in good agreement with those presented in Refs. [22]. This further validates the twisted SAFE-PML approach proposed in this paper.

\section{Numerical applications}

\subsection{Embedded helical wire}

One considers a steel helical wire buried in concrete with the same material characteristics as in Sec. 3 . The helix radius $R_{h}$ and step $L_{h}$ are respectively equal to $2 a$ and $46.9 a$. These values yield a helix lay angle of $\phi=15^{\circ}$, defined from the relation $\tan \phi=2 \pi R_{h} / L_{h}$. The torsion of the twisting system is $\tau a=0.134 a$. Inside a plane cut normal to the helix, the cross-section of the steel core is circular of radius $a$. In the $(x, y)$ twisting plane, note that this cross-section is not circular [6].

The interface position of the centered PML and the off-centered PML are set to $d=3.1 a$ and $d=1.1 a$ respectively. The origin $O^{\prime}$ of the off-centered PML lies on the helical axis of the wire (as in Fig. 1a). For both PMLs, the thickness and the averaged value of the absorbing function are: $h=0.9 a, \hat{\gamma}=2+4 i$.

Due to the relatively close correspondence of modes in a straight cylinder, one keeps the same labeling of modes as in Sec. 3.

\subsubsection{Validation of the off-centered PML}

Since the computational domain with a centered PML is quite large (see Fig. 2b), the off-centered configuration should be preferred to model an embedded helical wire (see Fig. 1b). The goal of this subsection is to check that the off-centered PML technique properly absorbs waves in a twisting system. Results are compared with those computed 


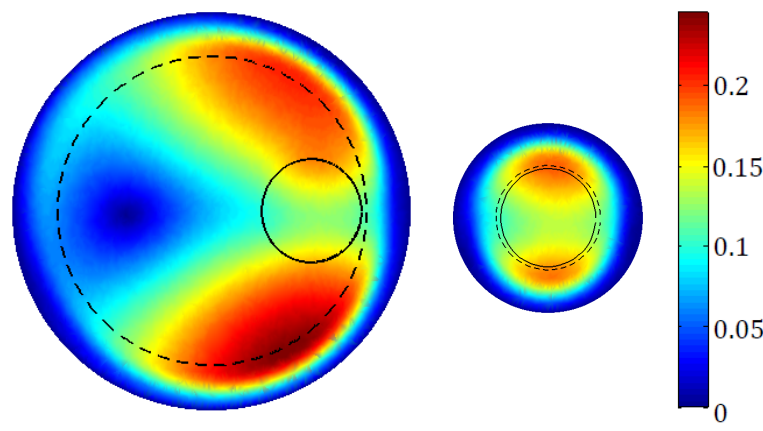

(a)

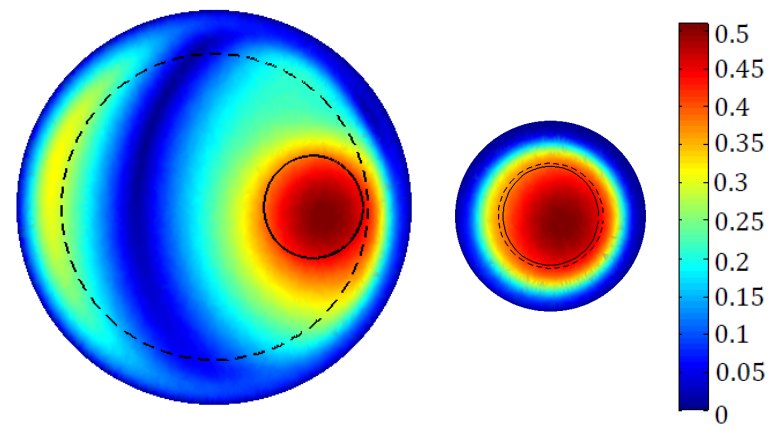

(b)

Figure 7: Modulus of displacement of the $L(0,1)$ mode in a) $y$ and b) $z$ directions at $\omega a / c_{s 0}=1.5$ computed from a centered PML (left) and an off-centered PML (right). Continuous line: steel-concrete interface, dashed line: PML interface.

with a centered PML method, which has been theoretically justified with twist in Sec. 2.4 and checked by numerical results in Sec. 3.

Figure 7 compares some displacement components of the $L(0,1)$ mode obtained from the centered and the offcentered PML at the dimensionless frequency $\omega a / c_{s 0}=1.5$. Finite elements are six-node triangles of averaged length $l_{e}=0.2 a$. The mode shapes inside the physical region, i.e. outside the PML, obtained by the off-centered PML are in good agreement with those of the centered PML. The axial wavenumbers $k a$ computed by both PMLs are also in agreement: $0.9718+0.1222 i$ for the centered PML and $0.9768+0.1218 i$ for the off-centered PML.

In this example, 5775 dofs (degrees of freedom) have been generated with the off-centered PML method against 21579 dofs with the centered PML. Another advantage of the off-centered PML technique is that the PML interface can be set closer to the core than with a centered PML, which allows to reduce numerical problems related to the transverse growth of leaky modes [32, 27, 23].

\subsubsection{Twist effects}

In this subsection, dispersion curves computed by the off-centered PML method are computed in order to highlight the influence of twist on the attenuation of leaky modes.

One focuses on $L(0, m)$ compressional modes, which are of interest for NDE. In cylinders, it has been shown that these modes have low axial attenuation and dispersion as well as high energy velocity [36, 37]. Such interesting properties occur when the $L(0, m)$ phase velocities are close to the longitudinal velocity $c_{l 0}$ of the core. In order to find these modes and avoid calculating too many radiation modes, the computation of eigenvalues can thus be centered around $\omega / c_{l 0}$. In this way, only 25 modes are computed at each frequency.

Figure 8 compares the dispersion curves for the energy velocity and the modal attenuation along the $z$ axis, computed for a helical and for a cylindrical wire. Note that a large frequency range must be considered to get the dispersion curves of higher order compressional modes (here, up to the $L(0,6)$ mode). The average length of finite elements has been set to $l_{e}=0.05 a$ in order to satisfy the meshing criterion $l_{e} \leq c_{s 0} / 5 f_{\max }$. About 82000 dofs have been generated. The number of dofs would have been tremendously larger with a centered PML.

Figure 8a shows that waves travel at slower energy velocity in the embedded helical wire than in a straight one. It should yet be noted that compressional modes remain faster than the other modes.

As shown in Fig. 8b, the axial attenuation of $L(0, m)$ modes is higher for the helical wire than for the straight one. This difference becomes more significant as the order of modes increases. This result shows that the twist of the helical geometry enhances the axial attenuation of modes. A similar effect has also been observed for twisted optical fibers [25] as well as for curved electromagnetic waveguides [12, 13].

This phenomenon can be confirmed by comparing the displacement of compressional modes in the helical and cylindrical wires. As an example, Fig. 9 gives the axial displacement of the $L(0,6)$ mode at its frequency of minimum attenuation $\left(\omega a / c_{s 0} \simeq 17.73\right)$. For the cylindrical wire, the displacement at the interface between the steel core and the 


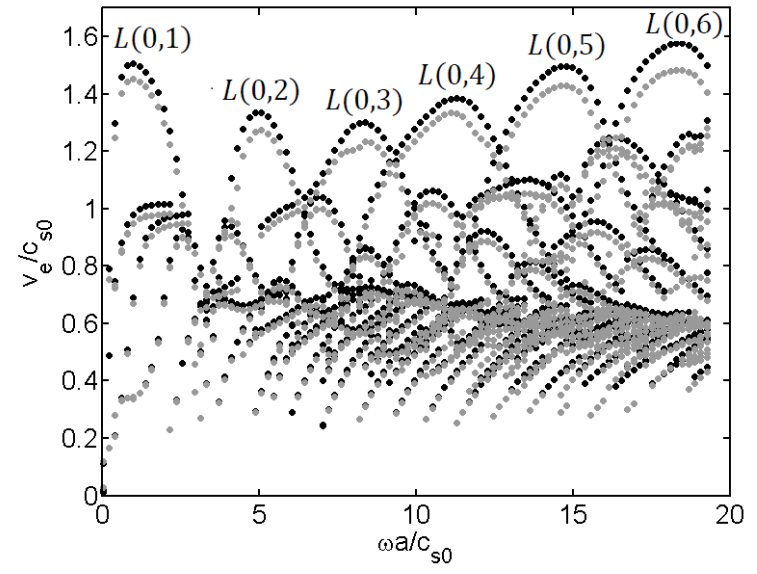

(a)

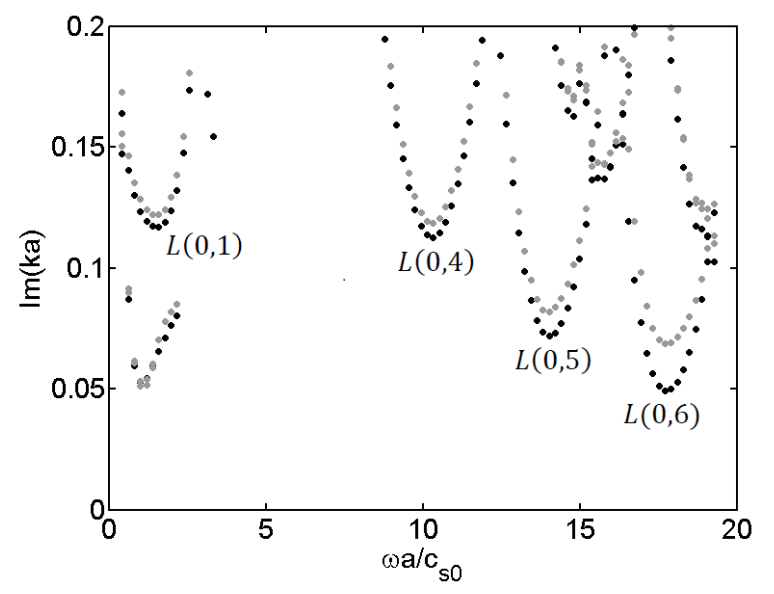

(b)

Figure 8: Dispersion curves for a) the energy velocity and b) the axial attenuation in an embedded cylinder (black) and in a helical wire with $\phi=15^{\circ}$ and $R_{h}=2 a$ (gray). $\rho_{\max }$ is set to 0.9 .

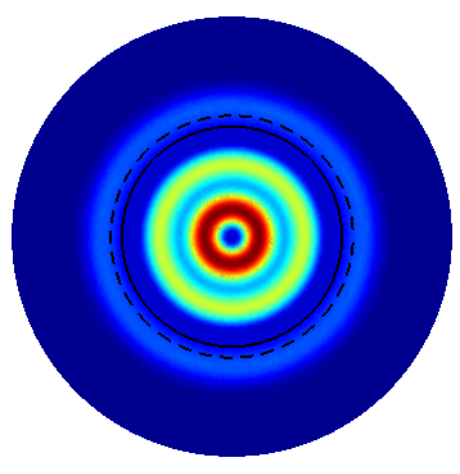

(a)

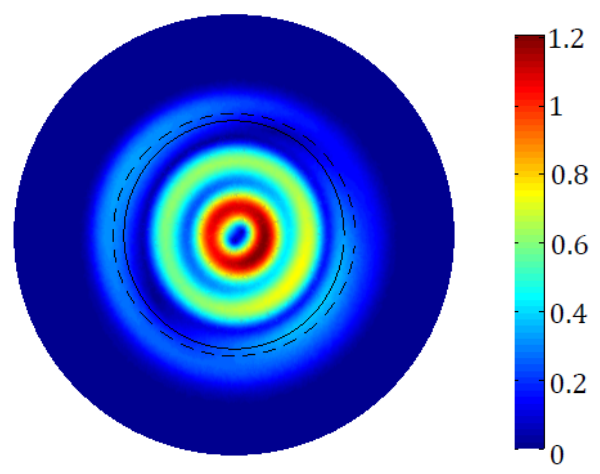

(b)

Figure 9: Modulus of the axial displacement of the $L(0,6)$ mode at $\omega a / c_{s 0}=17.73$ in an embedded a) cylinder and b) helical wire $\left(\phi=15^{\circ}, R_{h}=2 a\right)$. 


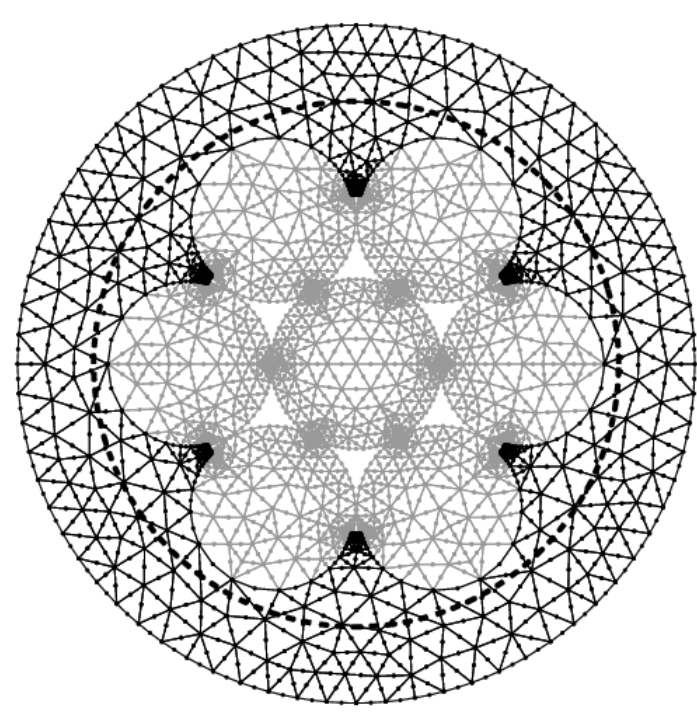

(a)

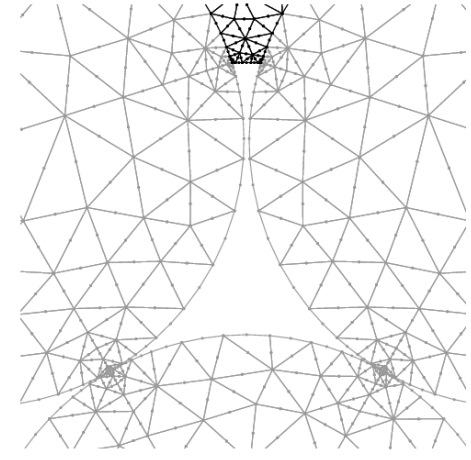

(b)

Figure 10: a) Cross-section FE mesh for an embedded seven wire strand $\left(\phi=7.9^{\circ}\right)$, b) zoom. Dashed line: PML interface.

surrounding medium almost vanishes, which explains why the attenuation of this mode is low. For the helical wire, the interface displacement turns out to be larger, and therefore the energy leakage is greater. As shown in Ref. [14], the same phenomenon occurs in curved plates.

These results show that the propagating distance of leaky modes in helical waveguides can be limited, which complicates the NDE of such structures.

\subsection{Embedded seven-wire strand}

One considers a steel seven-wire strand buried in concrete. The mechanical properties of steel are taken from Ref. [6] and are as follows: $E=2.17 e 11 \mathrm{~Pa}, v=0.28, \rho=7800 \mathrm{~kg} / \mathrm{m}^{3}, c_{l_{0}}=5963.7 \mathrm{~m} / \mathrm{s}, c_{s_{0}}=3296.6 \mathrm{~m} / \mathrm{s}$. The properties of concrete are the same as in Tab. 1. To model the embedded strand, a centered PML is used. The PML parameters are: $d=3.1 a, h=0.9 a$ and $\hat{\gamma}=2+4 i$. The cross-section of the structure, consisting of one central cylindrical wire surrounded by six peripheral helical wires, has been meshed using Gmsh [38] (see Fig. 10a). The central wire radius is $a=2.7 \mathrm{~mm}$. The helical wire radius is equal to $0.967 a$. The helix pitch is $L_{h}=240 \mathrm{~mm}$, yielding a helix radius $R_{h}=1.967 a$ and a lay angle $\phi=7.9^{\circ}$. The torsion of the twisting coordinate system is $\tau a=0.0705$.

As shown in Fig. 10b, there is no contact between peripheral wires. However, each peripheral wire has a contact point with the central wire. For simplicity, one assumes stick contact conditions (no slip, no separation and no friction are considered). The continuity of displacement is enforced at the six contact points as well as at the interface between the peripheral wires and the embedding medium (concrete). The region between two consecutive peripheral wires is filled with concrete up to a certain level. The region without concrete is considered as vacuum. For a better accuracy of numerical results, the mesh has been refined at contact points and in the concrete region between two consecutive peripheral wires, yielding 13233 dofs.

Figure 11 gives the dispersion curves for the energy velocity and the axial attenuation computed by the SAFEPML method. 150 modes have been calculated, centered around $+\omega / c_{l}$ (where $c_{l}$ is the bulk velocity of concrete). Comparing Fig. 11a with the results of Ref. [6] obtained for a free strand (i.e. in vacuum), the modal behavior is strongly modified due to the introduction of the surrounding medium. In addition to leakage effects, one points out that the surrounding medium has the other effect to make peripheral wires almost in contact (as can be seen in Fig. 10), which further modifies dispersion curves. 


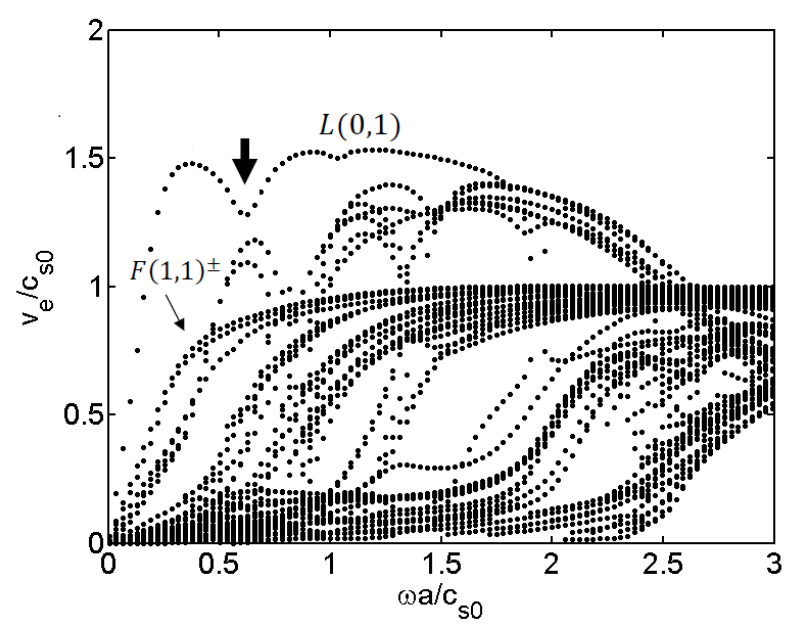

(a)

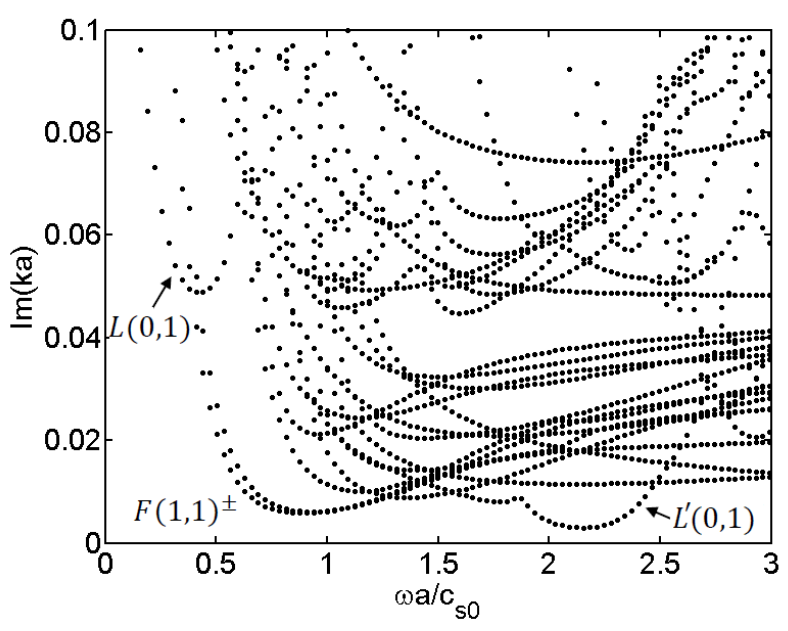

(b)

Figure 11: Dispersion curves for the embedded seven-wire strand for: a) the energy velocity and b) the axial attenuation. PML parameters are: $d=3.1 a, h=0.9 a$ and $\hat{\gamma}=2+4 i$. The arrow indicates the velocity drop of the $L(0,1)$ mode.

Although the modal behavior of the strand is far more complex than in a single wire, a fundamental compressional mode and a pair of flexural modes can be identified (by a visual inspection of mode shapes). These modes have a global motion and are labeled as $L(0,1)$ and $F(1,1)^{ \pm}$respectively. The notation \pm for flexural modes is used to point out the lack of axisymmetry of the structure, yielding distinct roots (instead of double roots with a cylinder).

For a free strand, it must be recalled that the $L(0,1)$ mode suffers a sudden energy velocity drop occuring at $\omega a / c_{s 0} \simeq 0.35$ (see Ref. [6,39]). This phenomenon is typical of seven-wire strands and is sometimes referred to as notch frequency in the literature [40]. As can be observed in Fig. 11a, the presence of concrete embedment seems to shift this velocity drop to a higher frequency $\left(\omega a / c_{s 0} \simeq 0.6\right)$ and this phenomenon is entirely described by a single continuous branch. Conversely, the velocity drop inside a free strand is the result of a curve veering phenomenon between two distinct branches [6].

From Fig. 11b, it is interesting to note that the flexural $F(1,1)^{ \pm}$and the so denoted $L^{\prime}(0,1)$ modes have the lowest attenuation in the frequency ranges $[0,1.5]$ and [2,2.5] respectively. Such modes could be useful for the NDE of embedded strands. As opposed to the global compressional $L(0,1)$ mode, the $L^{\prime}(0,1)$ mode is a compressional mode of local type. Its motion is essentially confined into the central wire, yielding low energy leakage into the surrounding medium.

\section{Conclusion}

In this work, twisted SAFE-PML methods have been applied to compute the eigenmodes of embedded helical structures. Two kinds of radial PML, centered and off-centered, have been implemented.

The centered PML method has been justified by theoretical considerations. It has also been validated by numerical tests perfomed on an arbitrarily twisted cylindrical bar. Results have shown that the eigenspectrum with twist is spread over a larger region than without twist. This phenomenon has been explained by the translation relation existing between the axial wavenumbers of the twisting and the untwisting systems.

For the modeling of a single embedded helical wire, an off-centered PML has been proposed. The advantage of this technique is that the PML interface is close to the wire cross-section. This allows to significantly reduce the computational domain compared to the centered PML technique and avoid numerical problems with the transverse growth of leaky modes. The off-centered PML method has been checked numerically from a comparison of results with those computed with the centered PML method. From a physical point of view, numerical results show that the 
twist of helical wires enhances the axial attenuation of modes compared to straight guides. This can make the NDE of helical waveguides more difficult.

Finally, the numerical study of an embedded seven-wire strand has shown that the dispersion curves are strongly modified in the presence of the surrounding medium. Numerical results have allowed to identify two global flexural modes and one local compressional modes that have the lowest attenuation in their respective frequency range. Such modes could be useful for the NDE of embedded strands.

\section{References}

[1] K. Jezzine, Approche modale pour la simulation globale de contrôles non-destructifs par ondes élastiques guidées (Modal approach for the full simulation of nondestructive tests by elastic guided waves, in french), Ph.D. thesis, Université Bordeaux (2006).

[2] I. Bartoli, A. Marzani, F. Lanza di Scalea, E. Viola, Modeling wave propagation in damped waveguides of arbitrary cross-section, Journal of Sound and Vibration 295 (2006) 685-707.

[3] T. Hayashi, W.-J. Song, J. L. Rose, Guided wave dispersion curves for a bar with an arbitrary cross-section, a rod and rail example, Ultrasonics 41 (3) (2003) 175-183.

[4] P. W. Loveday, Semi-analytical finite element analysis of elastic waveguides subjected to axial loads, Ultrasonics 49 (2009) 298-300

[5] F. Treyssède, Elastic waves in helical waveguides, Wave Motion 45 (2008) 457-470.

[6] F. Treyssède, L. Laguerre, Investigation of elastic modes propagating in multi-wire helical waveguides, Journal of Sound and Vibration 329 (2010) 1702-1716.

[7] M. J. S. Lowe, Plate waves for the NDT of diffusion bonded titanium, Ph.D. thesis, Imperial College London (1992).

[8] J. A. Simmons, E. Drescher-Krasicka, H. N. G. Wadley, Leaky axisymmetric modes in infinite clad rods .1, Journal of the Acoustical Society of America 92 (2) (1992) 1061-1090.

[9] E. A. J. Marcatili, Bends in optical dielectric guides, The Bell System Technical Journal 48 (7) (1969) 2013-2132.

[10] M. Miyagi, S. Kawakami, S. Nishida, Bending losses of a doubly clad slab waveguide, Optics communications 14 (1) (1975) $123-125$.

[11] N. N. Feng, G. R. Zhou, C. Xu, Computation of full-vector modes for bending waveguide using cylindrical perfectly matched layers, Journal of Lightwave Technology 20 (11) (2002) 1976-1980.

[12] K. R. Hiremath, M. Hammer, R. Stoffer, L. Prkna, J. Ctyroký, Analytic approach to dielectric optical bent slab waveguides, Optical and Quantum Electronics 37 (1-3) (2005) 37-61.

[13] B. M. A. Rahman, D. M. H. Leung, S. S. A. Obayya, K. T. V. Grattan, Numerical analysis of bent waveguides: bending loss, transmission loss, mode coupling, and polarization coupling, Optical Society of America 47 (16) (2008) 2961-2970.

[14] M. D. Beard, Guided wave inspection of embedded cylindrical structures, Ph.D. thesis, Imperial College London (2002).

[15] K. L. J. Fong, A study of curvature effects on guided elastic waves, Ph.D. thesis, Imperial College London (2005).

[16] L. E. Alsop, A. S. Goodman, G. Gregersen, Reflection and transmission of inhomogeneous waves with particular application to Rayleigh waves, Bulletin of the Seismological Society of America 64 (6) (1974) 1635-1652.

[17] A. C. Hladky-Hennion, P. Langlet, M. de Billy, Conical radiating waves from immersed wedges, Journal of the Acoustical Society of America 108 (6) (2000) 3079-3083.

[18] C. T. Schröder, W. R. Scott, On the complex conjugate roots of the Rayleigh equation: The leaky surface wave, Journal of the Acoustical Society of America 110 (6) (2001) 2867-2877.

[19] T. Vogt, M. Lowe, P. Cawley, The scattering of guided waves in partly embedded cylindrical structures, Journal of the Acoustical Society of America 113 (3) (2003) 1258-1272.

[20] M. Castaings, M. Lowe, Finite element model for waves guided along solid systems of arbitrary section coupled to infinite solid media, Journal of the Acoustical Society of America 123 (2) (2008) 696-708.

[21] Z. Fan, M. J. S. Lowe, M. Castaings, C. Bacon, Torsional waves propagation along a waveguide of arbitrary cross section immersed in a perfect fluid, Journal of the Acoustical Society of America 124 (4) (2008) 2002-2010.

[22] M. Mazzotti, I. Bartoli, A. Marzani, E. Viola, A coupled SAFE-2.5D BEM approach for the dispersion analysis of damped leaky guided waves in embedded waveguides of arbitrary cross-section, Ultrasonics 53 (7) (2013) 1227-1241.

[23] F. Treyssède, K. L. Nguyen, A.-S. Bonnet-BenDhia, C. Hazard, Finite element computation of trapped and leaky elastic waves in open stratified waveguides, accepted in Wave Motion.

[24] K. L. Nguyen, F. Treyssède, A.-S. Bonnet-BenDhia, C. Hazard, Computation of dispersion curves in elastic waveguides of arbitrary crosssection embedded in infinite solid media, 13th International Symposium on Nondestructive Characterization of Materials (NDCM-XIII), May 20-24 2013, Le Mans, France.

[25] A. Nicolet, F. Zolla, Y. Ould Agha, Leaky modes in twisted microstructured optical fibres, Waves in Random and Complex Media 17 (4) (2007) 1-16.

[26] Y. Ould Agha, F. Zolla, A. Nicolet, S. Guenneau, On the use of PML for the computation of leaky modes. An application to microstructured optical fibres, The International Journal for Computation and Mathematics in Electrical and Electronic Engineering 27 (1) (2008) $95-109$

[27] B. Goursaud, Étude mathématique et numérique de guides d'ondes ouverts non uniformes, par approche modale (Mathematical and numerical study of non uniform open waveguides, modal approach, in French), Ph.D. thesis, École Polytechnique (2010).

[28] A. Pelat, S. Felix, V. Pagneux, A coupled modal-finite element method for the wave propagation modeling in irregular open waveguides, Journal of the Acoustical Society of America 129 (3) (2011) 1240-1249.

[29] A.-S. Bonnet-BenDhia, B. Goursaud, C. Hazard, A. Prieto, Finite element computation of leaky modes in stratified waveguides, Ultrasonic Wave Propagation in Non Homogeneous Media, Springer Proceedings in Physics 128 (2009) 73-86.

[30] F. Treyssède, K. L. Nguyen, A.-S. Bonnet-BenDhia, C. Hazard, On the use of a SAFE-PML technique for modeling two-dimensional open elastic waveguides, Acoustics 2012, France (April 23-27 2012) 667-672. 
[31] R. Lehouq, D. Sorenen, C. Yang, ARPACK User's Guide : Solution of Large Scale Eignevalue Problems with Implicity Restarded Arnoldi Methods, SIAM Philadenphia, PA, 1998.

[32] S. Kim, J. E. Pasciak, The computation of resonances in open systems using a perfectly matched layer, Mathematics of Computation 78 (267) (2009) 1375-1398.

[33] F. Collino, P. Monk, The perfectly matched layer in curvilinear coordinates, Society for Insdustrial and Applied Mathematics 19 (6) (1998) 2061-2090.

[34] J. Zemanek, An experimental and theoretical investigation of elastic wave propagation in a cylinder, Journal of the Acoustical Society of America 51 (1972) 265-283.

[35] O. Onipede, S. B. Dong, Propagating waves and end modes in pretwisted beams, Journal of Sound and Vibration 195 (1996) $313-330$.

[36] B. Pavlakovic, M. Lowe, P. Cawley, High frequency low loss ultrasonic modes in embedded bars, Journal of Applied Mechanics 68 (2001) 67-75.

[37] M. D. Beard, M. J. S. Lowe, Non-destructive testing of rock bolts using guided ultrasonic waves, International Journal of Rock Mechanics and Mining Sciences 40 (2003) 527-536.

[38] C. Geuzaine, J.-F. Remacle, Gmsh: a three-dimensional finite element mesh generator with built-in pre- and post-processing facilities, International Journal for Numerical Methods in Engineering 79 (11) (2009) 1309-1331.

[39] F. Treyssède, A. Frikha, P. Cartraud, Mechanical modeling of helical structures accounting for translational invariance. Part 2: Guided wave propagation under axial loads, International Journal of Solids and Structure 50 (2013) 1383-1393.

[40] H. Kwun, K. A. Barlets, J. J. Hanley, Effects of tensile loading on the properties of elastic-wave propagation in a strand, Journal of the Acoustical Society of America 103 (6) (1998) 3370-3375. 